\title{
Synthesis of trans-1,2-diamines via sequential opening of cyclohexene oxide and aziridinium ions
}

\author{
Mariappan Periasamy,* Muthu Seenivasaperumal, Meduri Padmaja, \\ and Vutukuri Dharma Rao \\ School of Chemistry, University of Hyderabad, Central University P.O., \\ Hyderabad - 500 046, INDIA \\ E-mail: mpsc@uohyd.ernet.in
}
Dedicated to Professor P. T. Narasimhan for his contributions to chemistry on the occasion of his $75^{\text {th }}$ birthday
(received 28 Jan 04; accepted 19 May 04; published on the web 22 May 04)

\begin{abstract}
A synthesis of trans-1,2-diaminocyclohexane derivatives via opening of cyclohexene oxide with secondary amines followed by preparation and opening of the corresponding aziridinium ions in situ by primary and secondary amines is described. Use of chiral $\alpha$-methylbenzylamine for the opening of aziridinium ions gave a mixure of diastereomers that are readily separated by column chromatography. The $\mathrm{C}_{2}$ symmetric trans-1,2-bis (N-pyrrolidino)cyclohexane was resolved to obtain nonracemic samples that can be readily enriched by co-crystallization with fumaric acid to obtain enantiomerically pure compounds.
\end{abstract}

Keywords: 1,2-Amino alcohols, aziridinium ions, 1,2-diamines, resolution

\section{Introduction}

In recent years, chiral diamines have been widely used as ligands in asymmetric synthesis. ${ }^{1}$ In particular the salen complexes of chiral diamines have been used in several asymmetric transformations. ${ }^{2}$ Also, several biologically active entities are known to contain diamine moieties. ${ }^{3}$ For example, the $\mathrm{C}_{2}$ symmetric cyclohexyl diamines $\mathbf{1}$ and $\mathbf{2}$ have been reported to give $86 \%$ ee in asymmetric dihydroxylation of olefins using $\mathrm{OsO}_{4}{ }^{4}$ The diamine derivatives 3 and $\mathbf{4}$ are reported to have analgesics activity. ${ }^{5,6}$ We report here a convenient method of synthesis of the diamine system containing 1,2-cyclohexane moiety via sequential opening of cyclohexene oxide and aziridinium ion derivatives. 
<smiles>CN(C)[C@@H]1CCCC[C@H]1N(C)C</smiles>

1<smiles>C1CCC(N2CCCC[C@H]2N2CCCC2)C1</smiles>

2<smiles>CN(C(=O)Cc1ccc(Cl)c(Cl)c1)[C@@H]1CCCC[C@H]1N1CCCC1</smiles>

3<smiles>CN(C(=O)c1ccc(Cl)c(Cl)c1)[C@@H]1CCCC[C@H]1N1CCCC1</smiles>

4

\section{Results and Discussion}

In recent years, several procedures have been reported for the opening of cyclohexene oxide by primary and secondary amines to the corresponding trans amino alcohols. ${ }^{7,8}$ We have observed that the amino alcohols 6 can be readily prepared by refluxing cyclohexene oxide with the corresponding amine (Scheme 1). Preparation of the corresponding mesylates 7 is accomplished by the reaction of the amino alcohol with $\mathrm{MsCl}$ in the presence of triethylamine. Solvolysis of the mesylates 7 produces the aziridinium ions in situ that are readily trapped by carrying out the reaction in the presence of primary and secondary amines to obtain the required trans-diamines in $60 \%-80 \%$ yields (Table 1 ).

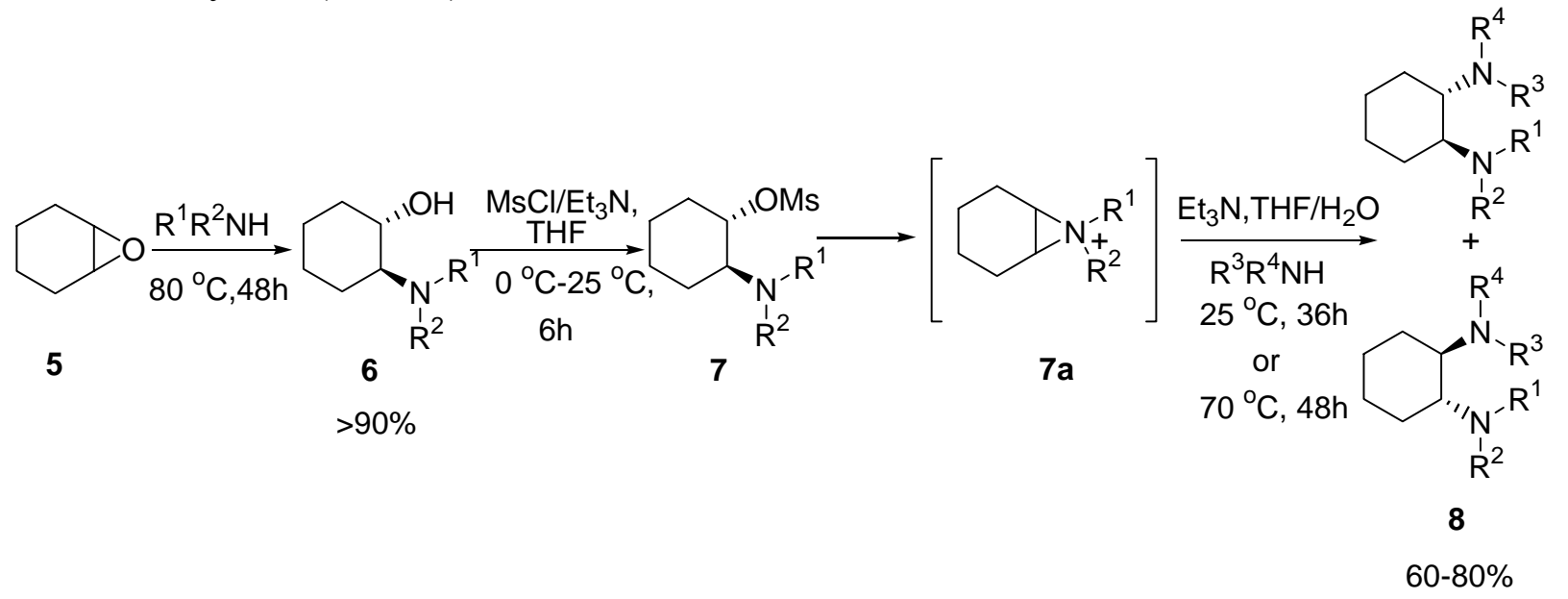

\section{Scheme 1}

Use of ammonia and methylamine in the reaction with the aziridinium ion 7a gave the corresponding diamines $\mathbf{8 a}$ and $\mathbf{8 b}$ in $70 \%$ yields (Entries 1 and 2, Table 1). Synthesis of racemic $\mathrm{C}_{2}$ symmetric amines $\mathbf{8 c}, \mathbf{8 d}$ and $\mathbf{8 e}$ is attained using the same secondary amines for the opening of the epoxide and the aziridinium ion (Entries 3, 4 and 5, Table 1). Reaction of the mesylate 7 using chiral $\alpha$-methylbenzylamine gave the diastereomeric mixtures of the diamines (8f, 8g) that can be readily separated by column chromatography. 
Table 1. Synthesis of trans-1,2-diaminocyclohexane derivatives by opening of aziridinium ion intermediates (Scheme 1)

S.No.

${ }^{a}$ The reaction were carried out using amino alcohol $(25 \mathrm{mmol}), \mathrm{MsCl}(30 \mathrm{mmol})$ and amine nucleophile (70 ml excess) at $25{ }^{\circ} \mathrm{C}$ for $48 \mathrm{~h}$. (Procedure A). (b) The reactions were carried out using amino alcohol $(10 \mathrm{mmol}), \mathrm{MsCl}(12 \mathrm{mmol})$ and amine neucleophile $(30 \mathrm{mmol})$ at $70{ }^{\circ} \mathrm{C}$ for 48h. (Procedure B). (c) The reactions were carried out using amino alcohol (10 mmol), Mscl $(12 \mathrm{mmol})$ and $\mathrm{R}-(+)-\alpha-$-methylbenzylamine $(10 \mathrm{mmol})$ at $25^{\circ} \mathrm{C}$ for $36 \mathrm{~h}$. (Procedure $\left.\mathrm{C}\right)$. (d) The yields are of the isolated product. The products were identified by $\mathrm{IR},{ }^{1} \mathrm{H},{ }^{13} \mathrm{C}$ NMR data and comparison with reported data. 
Table 2. Resolution of trans-1,2-bis ( $N$-pyrrolidino)cyclohexane 8c using dibenzoyl-L-tartaric acid and purification of non-racemic diamine using fumaric acid ${ }^{\mathrm{a}}$

\begin{tabular}{lcccccc}
\hline S.No & $\begin{array}{c}\text { Starting ee of the } \\
\text { diamine 8c }\end{array}$ & Diacid, & \multicolumn{3}{c}{ Diamine 8c from } \\
(mmol) & Precipitate & \multicolumn{2}{c}{ Filtrate } \\
\hline 1. & $00^{\mathrm{c}}$ & L (+) DBTA, 2 & $28(\mathrm{SS})$ & 70 & $77(\mathrm{RR})$ & 22 \\
2. & $00^{\mathrm{d}}$ & L (+) DBTA, 1 & $70(\mathrm{SS})$ & 36 & $37(\mathrm{RR})$ & 47 \\
3. & $77(\mathrm{RR})^{\mathrm{e}}$ & FA, 1.56 & $93(\mathrm{RR})$ & 60 & $33(\mathrm{RR})$ & 20 \\
4. & $93(\mathrm{RR})^{\mathrm{e}}$ & FA, 1.86 & $99(\mathrm{RR})$ & 75 & $40(\mathrm{RR})$ & 10 \\
\hline
\end{tabular}

${ }^{\mathrm{a}}$ The reactions were carried out using diamine $8 \mathrm{c}(2 \mathrm{mmol})$ and diacid in acetone at $25^{\circ} \mathrm{C}$ unless otherwise mentioned. All ee values reported here are based on the maximum $[\alpha]_{\mathrm{D}}^{25}=-31.85(\mathrm{C}$ $0.5,1 \mathrm{~N} \mathrm{HCl})$ obtained for the sample of $(R R)-8 \mathrm{c}$ with $>99 \%$ ee, $8 \mathrm{c}$ prepared following a reported procedure. ${ }^{4 \mathrm{~b}}$ The yields are of the isolated products, based on the total amount of the starting diamine used. ${ }^{\mathrm{b}} \mathrm{L}(+)$ DBTA $=$ dibenzoyl-L-tartaric acid, FA $=$ fumaric acid ${ }^{\mathrm{c}}$ The resolution was carried out in acetone solvent $(30 \mathrm{ml})$ for $12 \mathrm{~h} .{ }^{\mathrm{d}}$ The resolution was carried out in acetone solvent $(15 \mathrm{ml})$ for $20 \mathrm{~h} .{ }^{\mathrm{e}}$ The reaction was carried out in acetone solvent $(5 \mathrm{ml})$ for $12 \mathrm{~h}$.

We have also developed a procedure for the resolution of the $\mathrm{C}_{2}$ symmetric racemic diamine 8c. Non-racemic samples of 8 c with $28 \%-78 \%$ ee are readily obtained by resolution of this diamine with dibenzoyl-L-tartaric acid (Table 2). Enantiomerically pure samples are readily obtained from the nonracemic samples via preparation of the corresponding hydrogen bonded aggregates using fumaric acid (Table 2). ${ }^{9}$

\section{Conclusions}

The synthetic protocol described here adds to the 1,2-diamines that were previously prepared via opening of azidinium ions prepared in situ, ${ }^{10}$ trans-1,2-diamines containing cyclohexane moiety are generally synthesized using the corresponding aziridine derivatives. ${ }^{8 b, 11}$ These aziridine derivatives in turn need to be prepared following multi step synthesis. Accordingly, the procedures reported here for the preparation of trans-1,2-diamino cyclohexane moieties via opening of aziridinium ions in situ should make these derivatives more readily accessible. Since, several chiral diamines are useful as ligands in asymmetric synthesis ${ }^{1,2}$ and certain derivatives have proven applications in medicinal chemistry, ${ }^{5}$ the methods of synthesis of the diamine derivatives described here have good synthetic potential. 


\section{Experimental Section}

General Procedures. Cyclohexene Oxide, $\mathrm{MsCl}$, pyrrolidine, piperidine, Ammonia solution were purchased from commercial sources. THF solvent was freshly distilled over sodium/benzophenone under nitrogen. Optical rotations were measured in an AUTOPOL-II automatic polarimeter (readability \pm 0.01 ). Chromatography was carried out using Acme's silica gel (100-200 mesh) and neutral alumina. ${ }^{1} \mathrm{H}$ NMR $(200 \mathrm{MHz})$ and ${ }^{13} \mathrm{C}$ NMR $(50 \mathrm{MHz}) \mathrm{spectra}$ were recorded on Bruker AC-200 Spectrometer with $\mathrm{CDCl}_{3}$ as a solvent and TMS as reference $(\delta=0 \mathrm{ppm})$.

\section{Typical procedure for preparation of amino alcohols $6^{7}$}

A mixture of cyclohexene oxide $(50.6 \mathrm{ml}, 500 \mathrm{mmol})$ and pyrrolidine $(42 \mathrm{ml}, 500 \mathrm{mmol})$ was refluxed for $48 \mathrm{~h}$. The product was distilled under reduced pressure to obtain the pure trans- $( \pm)-2-$ (1-pyrrolidinyl)cyclohexanol 6. Yield: 80 g (94\%). B.p: $130{ }^{\circ} \mathrm{C} / 12 \mathrm{~mm} \mathrm{Hg}$; (Lit ${ }^{7}$ B.p.: $76{ }^{\circ} \mathrm{C} / 0.25 \mathrm{~mm} \mathrm{Hg}$.) IR (neat $\left.\mathrm{cm}^{-1}\right): 3452,2932,2858,1450,1078 .{ }^{1} \mathrm{H} \mathrm{NMR}\left(\mathrm{CDCl}_{3}\right): \delta 1.08-$ $1.39(\mathrm{~m}, 4 \mathrm{H}), 1.55-1.90(\mathrm{~m}, 7 \mathrm{H}), 2.00-2.19(\mathrm{~m}, 1 \mathrm{H}), 2.32-2.77(\mathrm{~m}, 5 \mathrm{H}), 3.20-3.39(\mathrm{~m}, 1 \mathrm{H}), 4.12$ $(\mathrm{s}, 1 \mathrm{H}) .{ }^{13} \mathrm{C} \mathrm{NMR}\left(\mathrm{CDCl}_{3}\right): \delta 21.2,23.6,24.2,25.4,33.3,47.2,64.9,70.7$.

\section{Typical procedure for preparation of trans-( \pm )-2-(1-pyrrolidinyl)cyclohexylamine $(8 \mathrm{Ba})^{12}$ (procedure A)}

Trans-( \pm )-2-(1-pyrrolidinyl)cyclohexanol $(25 \mathrm{mmol})$ was taken in dry THF $(100 \mathrm{ml})$ and triethylamine $(75 \mathrm{mmol})$ was added and the solution was cooled to $0{ }^{\circ} \mathrm{C}$. To this, methanesulfonyl chloride $(30 \mathrm{mmol})$ was added. The reaction mixture was allowed to stir at $25^{\circ} \mathrm{C}$ for $6 \mathrm{~h}$ and triethylamine $(50 \mathrm{mmol})$ was added. After stirring at $25^{\circ} \mathrm{C}$ for further $2 \mathrm{~h}$, ammonia solution $25 \%(71 \mathrm{ml})$ was added and the resulting two-phase reaction mixture was vigorously stirred. After $48 \mathrm{~h}$, the layers were separated and the aqueous layer was extracted with ether. The combined organic extract was washed with 5\% aqueous sodium hydrogen carbonate $(25 \mathrm{ml})$, water $(25 \mathrm{ml})$, dried over $\mathrm{MgSO}_{4}$ and evaporated. Distilled under reduced pressure to obtain the product.

\section{Typical procedure for preparation of trans $-( \pm)-1,2-b i s-\left(\right.$ pyrrolidino)cyclohexane $(8 c){ }^{4 b}$ (procedure B)}

Trans-( \pm )-2-(1-pyrrolidinyl)cyclohexanol $(1.69 \mathrm{~g}, 10 \mathrm{mmol})$ was taken in dry THF $(50 \mathrm{ml})$ and triethyl amine $(4.2 \mathrm{ml}, 30 \mathrm{mmol})$ was added and the solution was cooled to $0{ }^{\circ} \mathrm{C}$. To this, methanesulfonyl chloride $(0.94 \mathrm{ml}, 12 \mathrm{mmol})$ was added. The reaction mixture was allowed to stir at $25{ }^{\circ} \mathrm{C}$ for $6 \mathrm{~h}$ and triethyl amine $(2.78 \mathrm{ml}, 20 \mathrm{mmol})$ was added. After stirring at $25{ }^{\circ} \mathrm{C}$ for further $2 \mathrm{~h}$, pyrrolidine $(4.2,30 \mathrm{mmol})$ and water $(6 \mathrm{ml})$ was added and the resulting two phase mixture was refluxed for $48 \mathrm{~h}$. The aqueous and organic layers were separated and the aqueous layer was extracted with ether. The combined organic extract was washed with $5 \%$ aqueous 
sodium hydrogen carbonate $(15 \mathrm{ml})$, water $(15 \mathrm{ml})$, dried over $\mathrm{MgSO}_{4}$ and evaporated. The crude product was purified on silica gel column using hexane as eluent.

Spectral data for trans-( \pm )-2-(1-pyrrolidinyl)cyclohexylamine (8a). ${ }^{12}$ Yield: 70\%. B.p: $60{ }^{\circ} \mathrm{C} / 10 \mathrm{~mm} \mathrm{Hg}$; Colorless liquid. IR (neat $\left.\mathrm{cm}^{-1}\right): 3356,2961,2928,1448 .{ }^{1} \mathrm{H} \mathrm{NMR}\left(\mathrm{CDCl}_{3}\right): \delta$ 0.8-1.1 (m, 4H), 1.4-1.6 (m, 6H), 1.7-1.8 (d, 1H), 2.0-2.2 (t. 1H), 2.3-2.6 (m, 6H), 3.1 (br s, 2H). ${ }^{13} \mathrm{C} \mathrm{NMR}\left(\mathrm{CDCl}_{3}\right): \delta 21.5,23.6,24.9,25.3,34.6,47.0,52.6,64.9$.

trans-( \pm )-2-(1-Pyrrolidinyl)- $N$-methylcyclohexylamine (8b). ${ }^{11}$ Yield: $70 \%$. colorless liquid. IR (neat $\left.\mathrm{cm}^{-1}\right): 3315,2930,1448,1141 .{ }^{1} \mathrm{H}$ NMR $\left(\mathrm{CDCl}_{3}\right): \delta 0.9-1.0(\mathrm{~m}, 1 \mathrm{H}), 1.1-1.3(\mathrm{~m}, 4 \mathrm{H}), 1.5-$ $1.9(\mathrm{~m}, 6 \mathrm{H}), 2.1-2.3(\mathrm{~m}, 3 \mathrm{H}), 2.4(\mathrm{~s}, 3 \mathrm{H}), 2.5-2.7(\mathrm{~m}, 5 \mathrm{H}) .{ }^{13} \mathrm{C} \mathrm{NMR}\left(\mathrm{CDCl}_{3}\right): \delta 21.1,23.5,24.3$, 25.1, 30.9, 33.8, 46.6, 61.3, 61.9.

trans-( \pm )-1,2-Bis-(pyrrolidino)cyclohexane (8c). ${ }^{4 \mathrm{~b}}$ Yield: $80 \%$. colorless liquid. IR (neat cm ${ }^{-1}$ ): 2962, 2918, 1448. ${ }^{1} \mathrm{H}$ NMR $\left(\mathrm{CDCl}_{3}\right): \delta 1.2-1.8(\mathrm{~m}, 16 \mathrm{H}), 2.2-2.3(\mathrm{~m}, 2 \mathrm{H}), 2.5-2.6(\mathrm{~m}, 8 \mathrm{H}) .{ }^{13} \mathrm{C}$ $\operatorname{NMR}\left(\mathrm{CDCl}_{3}\right): \delta 21.3,23.4,25.2,51.4,63.1$.

trans-( \pm )-1,2-Bis-(piperidino)cyclohexane (8d). Yield: $75 \%$. colorless liquid. IR (neat cm ${ }^{-1}$ ): 2927, 1442, 1107. ${ }^{1} \mathrm{H}$ NMR $\left(\mathrm{CDCl}_{3}\right): \delta$ 0.9-1.85 (m, 20H), 2.2-2.3 (m, 2H), 2.5-2.7 (m, 8H). ${ }^{13} \mathrm{C}$ $\operatorname{NMR}\left(\mathrm{CDCl}_{3}\right): \delta 25.3,26.0,27.1,27.7,49.8,65.2$.

trans-( \pm )-1,2-Bis-(dimethlyamino)cyclohexane $\quad(8 \mathrm{e}){ }^{4} \quad$ Yield: $60 \%$. colorless liquid. IR $\left(\right.$ neat $\left.\mathrm{cm}^{1}\right): 2939,1458,1361 .{ }^{1} \mathrm{H}$ NMR $\left(\mathrm{CDCl}_{3}\right): \delta 1.1-1.3(\mathrm{~m}, 4 \mathrm{H}), 1.7-1.8(\mathrm{~m}, 2 \mathrm{H}), 1.9-2.0(\mathrm{~m}$, $2 \mathrm{H}), 2.4(\mathrm{~s}, 12 \mathrm{H}), 2.6(\mathrm{~m}, 2 \mathrm{H}) .{ }^{13} \mathrm{C} \mathrm{NMR}\left(\mathrm{CDCl}_{3}\right): \delta 23.7,24.4,40.0,63.9$.

Reaction of amino alcohol with $\mathrm{MsCl} \mathrm{Et}_{3} \mathrm{~N}$ followed by reaction with $\mathrm{Et}_{3} \mathrm{~N} /(\mathrm{R})-(+)-\alpha-$ methylbenzylamine $/ \mathrm{H}_{2} \mathrm{O}$ (Procedure $\mathrm{C}$ )

Amino alcohol $(10 \mathrm{mmol})$ was taken in dry THF $(50 \mathrm{ml})$ and triethylamine $(4.2 \mathrm{ml}, 30 \mathrm{mmol})$ was added and the solution was cooled to $0{ }^{\circ} \mathrm{C}$. To this, methanesulfonyl chloride $(0.94 \mathrm{ml}$, $12 \mathrm{mmol}$ ) was added. The reaction mixture was allowed to stir at $25{ }^{\circ} \mathrm{C}$ for $6 \mathrm{~h}$ and triethylamine $(2.78 \mathrm{ml}, 20 \mathrm{mmol})$ was added. After stirring at $25{ }^{\circ} \mathrm{C}$ for further $2 \mathrm{~h}$, (R)-(+)- $\alpha$ methylbenzylamine $(1.28 \mathrm{ml}, 10 \mathrm{mmol})$ and water $(6 \mathrm{ml})$ was added and the resulting two-phase reaction mixture was vigorously stirred. After $36 \mathrm{~h}$, the layers were separated and the aqueous layer was extracted with ether. The combined organic extract was washed with $5 \%$ aqueous sodium hydrogen carbonate $(15 \mathrm{ml})$, water $(15 \mathrm{ml})$, dried over $\mathrm{MgSO}_{4}$ and evaporated. The crude product was purified on neutral alumina column using hexane:ethyl acetate (99:1) mixture as eluent.

Spectral data for trans-(-)-2-(1-pyrrolidinyl)- $\boldsymbol{N}$-(R)-phenylethylcyclohexylamine (8f). Yield: 0.95g (35\%). IR (KBr cm $\left.{ }^{-1}\right): 3275,3059,3026,2930,2860,1601,1494,1448,1132,758,698$. ${ }^{1} \mathrm{H}$ NMR $\left(\mathrm{CDCl}_{3}\right): \delta$ 0.7-1.2 (m, 4H), $1.3(\mathrm{~d}, \mathrm{~J}=6.48 \mathrm{~Hz}, 3 \mathrm{H}), 1.4-1.9(\mathrm{~m}, 8 \mathrm{H}), 2.2-2.8(\mathrm{~m}, 6 \mathrm{H}), 2.99(\mathrm{bs}$, $1 \mathrm{H}), 3.7$ (q, J = 6.4 Hz, 1H), 7.1-7.5 (m, 5H). ${ }^{13} \mathrm{C} \mathrm{NMR}\left(\mathrm{CDCl}_{3}\right): \delta 21.9,24.0,24.5,25.2,33.2,47.2,57.5$, 59.6, 62.3, 126.4, 126.6, 128.1, 148.0. $[\alpha]_{\mathrm{D}}^{25}$ : - $48.9\left(\mathrm{C} \mathrm{0.49,} \mathrm{CHCl}_{3}\right) . \mathrm{MS}(\mathrm{EI}): \mathrm{m} / \mathrm{z} 272\left(\mathrm{~m}^{+}\right)$. Analysis calculated for $\mathrm{C}_{18} \mathrm{H}_{28} \mathrm{~N}_{2}$ : C, 79.41; H, 10.29; N, 10.29. Found: C, 79.37; H, 10.39; N, 10.28.

trans-(+)-2-(1-Pyrrolidinyl)- $\mathbf{N}$-(R)-phenylethylcyclohexylamine (8f). Yield: $0.95 \mathrm{~g}$ (35\%). IR

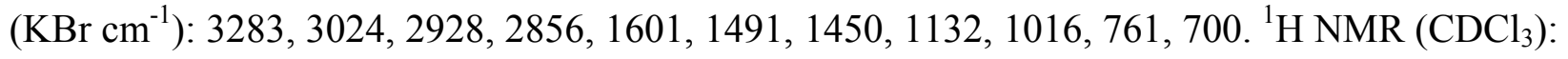


$\delta$ 0.9-1.3 (m, 4H), $1.4(\mathrm{~d}, \mathrm{~J}=6.4 \mathrm{~Hz}, 3 \mathrm{H}), 1.4-1.8(\mathrm{~m}, 8 \mathrm{H}), 1.8-2.5(\mathrm{~m}, 6 \mathrm{H}), 2.8(\mathrm{bs}, 1 \mathrm{H}), 3.7(\mathrm{q}, \mathrm{J}$ $=6.4 \mathrm{~Hz}, 1 \mathrm{H}), 7.1-7.4(\mathrm{~m}, 5 \mathrm{H}) .{ }^{13} \mathrm{C} \mathrm{NMR}\left(\mathrm{CDCl}_{3}\right): \delta 21.6,23.6,24.5,24.7,25.4,31.6,47.0$,

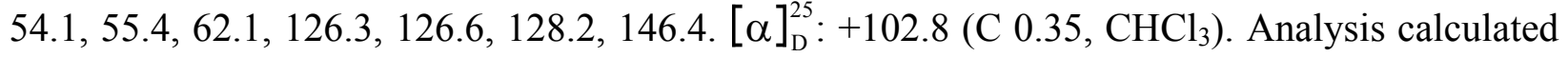
for $\mathrm{C}_{18} \mathrm{H}_{28} \mathrm{~N}_{2}$ : C, 79.41; H, 10.29; N, 10.29. Found: C, 79.12; H, 10.35; N, 10.32.

trans-(-)-2-(1- $N, N$-Diethylamino)- $N$-(R)-phenylethylcyclohexylamine $\quad$ (8g). Yield: $\quad 0.01 \mathrm{~g}$ (37\%). IR ( $\left.\mathrm{KBr} \mathrm{cm}^{-1}\right): 3290,3018,2964,1452,1369,758,696 .{ }^{1} \mathrm{H} \mathrm{NMR}\left(\mathrm{CDCl}_{3}\right): \delta 1.0-1.1(\mathrm{t}$, $6 \mathrm{H}), 1.3-1.4(\mathrm{~d}, 3 \mathrm{H}), 1.4-1.8(\mathrm{~m}, 4 \mathrm{H}), 2.2-2.4(\mathrm{~m}, 6 \mathrm{H}), 2.5-2.7(\mathrm{~m}, 4 \mathrm{H}), 2.9$ (br s, 1H), 3.7-3.8 (q, 1H), 7.1-7.4 (m, 5H). ${ }^{13} \mathrm{C} \mathrm{NMR}\left(\mathrm{CDCl}_{3}\right): \delta 15.1,23.6,24.3,24.9,26.0,33.8,43.3,57.8,58.6$, 63.9, 126.4, 127.0, 128.1, 148.0. $[\alpha]_{\mathrm{D}}^{25}$ : - $63.7\left(\mathrm{C} \mathrm{1.35,} \mathrm{CHCl}_{3}\right) . \mathrm{MS}(\mathrm{EI}): \mathrm{m} / \mathrm{z} 274\left(\mathrm{~m}^{+}\right)$.

trans-(+)-2-(1- $\mathbf{N}, \boldsymbol{N}$-Diethylamino)- $\boldsymbol{N}$-(R)-phenylethylcyclohexylamine $\quad$ (8g). Yield: $1.04 \mathrm{~g}$ (38\%). IR ( $\left.\mathrm{KBr} \mathrm{cm}^{-1}\right): 3290,3018,2964,1452,1369,758,696 .{ }^{1} \mathrm{H} \mathrm{NMR}\left(\mathrm{CDCl}_{3}\right): \delta 0.9-1.1(\mathrm{t}$, $6 \mathrm{H}), 1.3-1.4(\mathrm{~d}, 3 \mathrm{H}), 1.5-1.7(\mathrm{~m}, 4 \mathrm{H}), 1.9-2.4(\mathrm{~m}, 10 \mathrm{H}), 3.0($ br s, $1 \mathrm{H}), 3.7-3.8(\mathrm{q}, 1 \mathrm{H}), 7.1-$ 7.3(m, 5H). ${ }^{13} \mathrm{C} \mathrm{NMR}\left(\mathrm{CDCl}_{3}\right): \delta 14.6,23.2,24.5,26.0,31.6,42.6,53.7,54.0,63.7,126.4$, 126.5, 128.1, 148.0. $[\alpha]_{\mathrm{D}}^{25}:+92.3\left(\mathrm{C} 0.39, \mathrm{CHCl}_{3}\right)$.

Typical procedure for resolution of trans-( \pm )-1,2-bis-(pyrrolidino)cyclohexane (8c) using dibenzoyl-L-tartaric acid (Table 2) ${ }^{9}$

The trans- $( \pm)-1,2-b i s($ pyrrolidino)cyclohexane 8c $(2 \mathrm{mmol}, 0.44 \mathrm{~g})$ was added to the dibenzoyl-Ltartaric acid $(2 \mathrm{mmol}, 0.71 \mathrm{~g})$ dissolved in acetone $(30 \mathrm{ml})$ and stirred at $25{ }^{\circ} \mathrm{C}$ for $12 \mathrm{~h}$. The precipitate obtained was filtered and decomposed by stirring with $10 \%$ aq. $\mathrm{NaOH}$ and the free diamine was extracted with ether. The organic extract was washed with brine, dried over anhydrous $\mathrm{Na}_{2} \mathrm{SO}_{4}$, and evaporated to obtain the (SS)-8c diamine, yield: $0.16 \mathrm{~g}, 36 \%$ and $70 \%$ ee. The filtrate was evaporated to dryness and decomposed using $10 \%$ aq. $\mathrm{NaOH}$. After workup, the (RR)-8c diamine was isolated, yield: $0.2 \mathrm{~g}, 47 \%$ and $37 \%$ ee.

Typical procedure for enrichment of the non-racemic trans-( \pm )-1,2-bis(pyrrolidino)cyclohexane (8c) using fumaric acid (Table 2) ${ }^{9}$

Fumaric acid (1.56mmol, $0.18 \mathrm{~g})$ was added to the non-racemic trans -1,2-bis (pyrrolidino)cyclohexane (78\% (RR)-8c 2mmol, 0.44g) dissolved in acetone (5ml) and stirred at $25{ }^{\circ} \mathrm{C}$ for $12 \mathrm{~h}$. The precipitate obtained was filtered and decomposed by stirring with $10 \%$ aq. $\mathrm{NaOH}$. The free diamine was extracted with ether. The combined organic extract was washed with brine, dried over anhydrous $\mathrm{Na}_{2} \mathrm{SO}_{4}$, and evaporated to obtain the (RR)-8c diamine, yield: $0.26 \mathrm{~g}, 60 \%$, and $93 \%$ ee. The filtrate was evaporated to dryness and decomposed using $10 \%$ aq. $\mathrm{NaOH}$. After workup, the (RR)-8c diamine was obtained, yield: $0.1 \mathrm{~g}, 20 \%$ and $33 \%$ ee.

\section{Acknowledgements}

We thank the CSIR and DST (New Delhi) for financial support. We are also grateful to the UGC (New Delhi) for support under the "University of Potential for Excellence" program. 


\section{References}

1. (a) Bennani, Y. L.; Hanessian, S. Chem. Rev. 1997, 97, 3161. (b) O’Brien, P. J. Chem. Soc., Perkin Trans. 1 1998, 1439. (c) Lucet, D.; Le Gall T.; Mioskowski, C. Angew. Chem., Int. Ed. 1998, 37, 2580. (d) O’Brien, P. J. Chem. Soc., Perkin Trans. 1 1998, 1439. (e) Bhuniya, D.; Dattagupta, A.; Singh, V. K. J. Org. Chem. 1996, 61, 6108.

2. (a) Zang, W.; Loebach, L. J.; Wilson, S.R.; Jacobsen, E. N. J. Am. Chem. Soc. 1990, 112, 2801. (b) Jacobsen, E. N.; Zang, W.; Muci, A. R.; Ecker, J.R.; Deng, L. J. Am. Chem. Soc. 1991, 113, 7063. (c) Deng, L.; Jacobsen, E. N. J. Org. Chem. 1992, 57, 4320. (d) Katsuki, T. Coord. Chem. Rev. 1995, 140, 189. (e) Canali, L.; Sherrington, D. C. Chem. Soc. Rev. 1999, $28,85$.

3. Chang, A. C.; Takemori, A. E.; Ojala, W. H.; Gleason, W. B.; Portoghese, P. S. J. Med. Chem. 1994, 37, 4490.

4. (a) Tolkes, M.; Snyder, J. K. Tetrahedron Lett. 1986, 27, 3951. (b) Corey, E. J.; Sarshar, S.; Azimioara, M. D.; Newbold, R. C.; Noe, M. C. J. Am. Chem. Soc. 1996, 118, 7851.

5. Szmuszkovicz, J.; Von Voigtlander, P. F. J. Med. Chem. 1982, 25, 1125.

6. Cheney, B. V.; Szmuszkovicz, J.; Lahti, R. A.; Zichi, D. A. J. Med. Chem. 1985, 28, 1853.

7. Radesca, L.; Bowen, W. D.; Paolo, L. D.; De Costa, B. R. J. Med. Chem. 1991, 34, 3058.

8. (a)Sekar, G.; Singh, V. K. J. Org. Chem. 1999, 64, 287. (b) Bisai, A.; Pandey, G.; Pandey, M. K.; Singh, V. K. Tetrahedron Lett. 2003, 44, 5839. (c) Barbaro, P.; Bianchini, C.; Sernau, V. Tetrahedron:Asymmetry 1996, 7, 843. (d) Chini, M.; Crotti, P.; Favero, L.; Macchia, F.; Pineschi, M. Tetrahedron Lett. 1993, 34, 433. (e) Kato, K.; Furuta, K.; Yamamoto, H. Synlett 1992, 565.

9. Periasamy, M.; Sivakumar, S.; Narsireddy, M.; Padmaja, M. Org. Lett. 2004, 6, 265.

10. (a) Saravanan, P.; Singh, V. K. Tetrahedron Lett. 1998, 39, 167. (b) De Sousa, S. E.; O’Brien, P.; Poumellec, P. J. Chem. Soc., Perkin Trans. 1 1998, 1483. (c) O’Brien, P.; Poumellec, P. Tetrahedron Lett. 1996, 37, 5619. (d) De Sousa, S. E.; O’Brien, P.; Poumellec, P. Tetrahedron:Asymmetry 1997, 8, 2613. (e) Anderson, S. R.; Ayers, J. T.; De Vries, K. M.; Ito, F.; Mendenhall, D.; Vanderplas, B. C. Tetrahedron:Asymmetry 1999, 10, 2655. (f) Liu, Q.; Marchington, A. P.; Boden, N.; Rayner, C. M. J. Chem. Soc., Perkin Trans. 1 1997, 511. (g) De Sousa, S. E.; O’Brien, P.; Steffens, H. C. Tetrahedron Lett. 1999, 40, 8423. (h) Colman, B.; De Sousa, S. E.; O’Brien, P.; Towers, T. D.; Watson, W. Tetrahedron:Asymmetry 1999, 10, 4175.

11. (a) De Costa, B.; George, C.; Rothman, R. B.; Jacobson, A. E.; Rice, K. C. FEBS Lett. 1987, 223, 335. (b) Sekar, G.; Singh, V. K. J. Org. Chem. 1999, 64, 2537.

12. De Costa, B. R.; Radesca, L. Heterocycles 1990, 31, 1837. 